\title{
FINITE GROUPS WITH THE SAME JOIN GRAPH AS A FINITE NILPOTENT GROUP
}

\author{
ANDREA LUCCHINI \\ Dipartimento di Matematica "Tullio Levi-Civita”, Università degli Studi di Padova, \\ Via Trieste 63, 35121 Padova, Italy \\ e-mail:lucchini@math.unipd.it
}

(Received 30 March 2020; revised 22 June 2020; accepted 24 July 2020; first published online 17 August 2020)

\begin{abstract}
Given a finite group $G$, we denote by $\Delta(G)$ the graph whose vertices are the proper subgroups of $G$ and in which two vertices $H$ and $K$ are joined by an edge if and only if $G=\langle H, K\rangle$. We prove that if there exists a finite nilpotent group $X$ with $\Delta(G) \cong \Delta(X)$, then $G$ is supersoluble.
\end{abstract}

2010 Mathematics Subject Classification. 20D60, 20D30

1. Introduction. Let $G$ be a finite group. We define a graph $\Delta(G)$ as follows. The vertices of $\Delta(G)$ are the proper subgroups of $G$. Two vertices $H$ and $K$ are joined by an edge if $G$ is generated by $H$ and $K$, that is, $G=\langle H, K\rangle$. This graph was introduced in [1] and is called the join graph of $G$. We have slightly modified the original definition, including in the vertex set the subgroups of $G$ contained in the Frattini subgroup $\operatorname{Frat}(G)$ of $G$. They correspond to isolated vertices of $\Delta(G)$. In particular, $\Delta(G)$ contains no edge if $G$ is cyclic of prime-power order.

A typical question that arises whenever a graph is associated with a group is the following:

QuESTION 1. How similar are the structures of two finite groups $G_{1}$ and $G_{2}$ if the graphs $\Delta\left(G_{1}\right)$ and $\Delta\left(G_{2}\right)$ are isomorphic?

We will say that a subgroup $H$ of $G$ is a maximal intersection in $G$ if there exists a family $M_{1}, \ldots, M_{t}$ of maximal subgroups of $G$ with $H=M_{1} \cap \cdots \cap M_{t}$. Let $\mathcal{M}(G)$ be the subposet of the subgroup lattice of $G$ consisting of $G$ and all the maximal intersections in $G$. Notice that $\mathcal{M}(G)$ is a lattice in which the meet of two elements $H$ and $K$ coincides with their intersection and their join is the smallest maximal intersection in $G$ containing $\langle H, K\rangle$ (in general $\langle H, K\rangle$ is not a maximal intersection, see the example at the end of Section 2). The maximum element of $\mathcal{M}(G)$ is $G$, and the minimum element coincides with the Frattini subgroup $\operatorname{Frat}(G)$ of $G$. The role played by $\mathcal{M}(G)$ in investigating the property of the graph $\Delta(G)$ is clarified by the following proposition.

Proposition 2. The lattice $\mathcal{M}(G)$ can be completely determined from the knowledge of the graph $\Delta(G)$. In particular, if $G_{1}$ and $G_{2}$ are finite groups and the graphs $\Delta\left(G_{1}\right)$ and $\Delta\left(G_{2}\right)$ are isomorphic, then also the lattices $\mathcal{M}\left(G_{1}\right)$ and $\mathcal{M}\left(G_{2}\right)$ are isomorphic.

Notice that the condition $\mathcal{M}\left(G_{1}\right) \cong \mathcal{M}\left(G_{2}\right)$ is necessary but not sufficient to ensure $\Delta\left(G_{1}\right) \cong \Delta\left(G_{2}\right)$. For example, consider $G_{1}=A \times\langle x\rangle$ and $G_{2}=\operatorname{Sym}(3) \times\langle y\rangle$, where $A \cong$ $C_{3} \times C_{3},\langle x\rangle \cong C_{2}$ and $\langle y\rangle \cong C_{3}$. Let $a_{1}, a_{2}, a_{3}, a_{4}$ and $b_{1}, b_{2}, b_{3}, b_{4}$ be generators for 
the four different non-trivial proper subgroups of, respectively, $A$ and $\operatorname{Sym}(3)$. The map sending $A$ to $\operatorname{Sym}(3)$ and $\left\langle a_{i}, x\right\rangle$ to $\left\langle b_{i}, y\right\rangle$ for $1 \leq i \leq 4$ induces an isomorphism between $\mathcal{M}\left(G_{1}\right)$ and $\mathcal{M}\left(G_{2}\right)$; however, all the subgroups of $G_{1}$ are maximal intersections, while $\langle(1,2,3) y\rangle$ and $\left\langle(1,2,3) y^{2}\right\rangle$ are not maximal intersections in $G_{2}$. In particular, $\Delta\left(G_{1}\right)$ has 12 vertices and $\Delta\left(G_{2}\right)$ has 14 vertices. So the following variation of Question 1 arises.

Question 3. How similar are the structures of two finite groups $G_{1}$ and $G_{2}$ if the lattices $\mathcal{M}\left(G_{1}\right)$ and $\mathcal{M}\left(G_{2}\right)$ are isomorphic?

Our aim is to start to investigate Questions 1 and 3, considering the particular case when $G_{1}$ is a finite nilpotent group. Notice that if $G_{1}$ is a finite nilpotent group and $\Delta\left(G_{1}\right) \cong$ $\Delta\left(G_{2}\right)$, then $G_{2}$ is not necessarily nilpotent. For example, if $p$ is an odd prime, $C_{p}$ is the cyclic group of order $p$, and $D_{2 p}$ is the dihedral group of order $2 p$, then the subgroup lattices of $C_{p} \times C_{p}$ and $D_{2 p}$ are isomorphic and therefore $\Delta\left(C_{p} \times C_{p}\right) \cong \Delta\left(D_{2 p}\right)$. Our main result is the following.

THEOREM 4. Let $G$ be a finite group. If there exists a finite nilpotent group $X$ with $\mathcal{M}(G) \cong \mathcal{M}(X)$, then $G$ is supersoluble.

COROLLARY 5. Let $G$ be a finite group. If there exists a finite nilpotent group $X$ with $\Delta(G) \cong \Delta(X)$, then $G$ is supersoluble.

Let $\mathfrak{M}$ be the family of the finite groups $G$ with the property that $\mathcal{M}(G) \cong \mathcal{M}(X)$ for some finite nilpotent group $X$. In a similar way, let $\mathfrak{D}$ be the family of the finite groups $G$ with the property that $\Delta(G) \cong \Delta(X)$ for some finite nilpotent group $X$. By Theorem 4 , if $G \in \mathfrak{M}$, then $G$ is supersoluble, but there exist supersoluble groups which do not belong to $\mathfrak{M}$ and it is not easy to give a complete characterization of the finite groups in $\mathfrak{M}$ or in $\mathfrak{D}$. We give a solution of this problem in the particular case when $G$ is a finite group with $\operatorname{Frat}(G)=1$. Recall that a finite group $G$ is called a $P$-group of $G$, it is either a non-cyclic elementary abelian group or a semidirect product of an elementary abelian $p$-group $A$ by a group of prime order $q \neq p$ which induces a non-trivial power automorphism on $A$ (in particular each subgroup of $A$ is normal in $G$ ). Some of the properties of $P$-groups that will be used throughout the paper are highlighted in [17, Section 2.2].

Proposition 6. Let $G$ be a finite group with $\operatorname{Frat}(G)=1$. Then, $G \in \mathfrak{D}$ if and only if $G$ is a direct product of groups with pairwise coprime orders that are either P-groups or elementary abelian p-groups.

The classification of the Frattini-free groups in $\mathfrak{M}$ is more difficult. First, we need a definition. Let $t \geq 2$ be a positive integer and $p_{1}, \ldots, p_{t}$ be prime numbers with the property that $p_{i+1}$ divides $p_{i}-1$ for $1 \leq i \leq t-1$. We denote by $\Lambda\left(p_{1}, \ldots, p_{t}\right)$ the set of the direct products $H_{1} \times \cdots \times H_{t-1}$, where $H_{i} \cong C_{p_{i}}^{n_{i}} \rtimes C_{p_{i+1}}$ is a non-abelian $P$-group. Moreover, we will denote by $\Lambda^{*}\left(p_{1}, \ldots, p_{t}\right)$ the direct products $X \times Y$ with $X \in \Lambda\left(p_{1}, \ldots, p_{t}\right)$ and $Y \cong C_{p_{1}}$. Finally, let $\Lambda$ (respectively $\left.\Lambda^{*}\right)$ be the union of all the families $\Lambda\left(p_{1}, \ldots, p_{t}\right)$ (respectively, $\Lambda^{*}\left(p_{1}, \ldots, p_{t}\right)$ ), for any possible choice of $t$ and $p_{1}, \ldots, p_{t}$.

Proposition 7. Let $G$ be a finite group with $\operatorname{Frat}(G)=1$. Then, $G \in \mathfrak{M}$ if and only if $G$ is a direct product $H_{1} \times \cdots \times H_{u}$, where the orders of the factors are pairwise coprime and each of the factors is of one of the following types:

(1) an elementary abelian p-group;

(2) a group in $\Lambda$;

(3) a group in $\Lambda^{*}$. 
It follows from the previous proposition that $\operatorname{Sym}(3) \times C_{2}$ is an example (indeed the one of smallest possible order) of a supersoluble group which does not belong to $\mathfrak{M}$.

Notice that our proof of Theorem 4 uses the classification of the finite simple groups. Theorem 4 is invoked in the proof of Proposition 7, which therefore in turn depends on the classification. On the contrary, Proposition 6 can be directly proved without using Theorem 4 and the classification of the finite simple groups. Indeed, it turns out that if $G \in \mathfrak{D}$ and Frat $(G)=1$, then $G$ has the same subgroup lattice as a finite abelian group, and the groups with this property have been classified by Baer [3]. However, we are not able to deduce Corollary 5 from Proposition 6, so also our proof of this result depends on the classification. To avoid the use of the classification in the proof of Corollary 5, one should give a positive answer to the following question that we leave open.

QUESTION 8. Does $\Delta\left(G_{1}\right) \cong \Delta\left(G_{2}\right)$ imply $\Delta\left(G_{1} / \operatorname{Frat}\left(G_{1}\right)\right) \cong \Delta\left(G_{2} / \operatorname{Frat}\left(G_{2}\right)\right)$ ?

The obstacle in dealing with this question is that it is not clear whether and how one can deduce which vertices of the graph $\Delta(G)$ correspond to subgroups of $G$ containing $\operatorname{Frat}(G)$.

2. Preliminary results. Denote by $\mathcal{N}_{G}(X)$ the neighborhood of the vertex $X$ in the graph $\Delta(G)$. We define an equivalence relation $\equiv_{G}$ by the rules $X \equiv_{G} Y$ if and only if $\mathcal{N}_{G}(X)=\mathcal{N}_{G}(Y)$. If $X \leq G$, let $\tilde{X}$ be the intersection of the maximal subgroups of $G$ containing $X$ (setting $\tilde{G}=G$ ). $\tilde{X}=\tilde{Y}$.

LEMMA 9. $\mathcal{N}_{G}(X) \subseteq \mathcal{N}_{G}(Y)$ if and only if $\tilde{X} \leq \tilde{Y}$. In particular, $X \equiv_{G} Y$ if and only if

Proof. Assume $\mathcal{N}_{G}(X) \subseteq \mathcal{N}_{G}(Y)$ and let $M$ be a maximal subgroup of $G$. If $Y \leq M$, then $\langle Y, M\rangle \neq G$, so $M \notin \mathcal{N}_{G}(Y)$. It follows that $M \notin \mathcal{N}_{G}(X)$, that is, $\langle X, M\rangle \neq G$. This implies $X \leq M$. It follows that $\tilde{X} \leq \tilde{Y}$. Conversely, assume $\tilde{X} \leq \tilde{Y}$, or equivalently that every maximal subgroup of $G$ containing $Y$ contains also $X$. If $Z \notin \mathcal{N}_{G}(Y)$, then $\langle Y, Z\rangle \leq M$ for some maximal subgroup $M$ of $G$. It follows $\langle X, Z\rangle \leq M$ and consequently $Z \notin \mathcal{N}_{G}(X)$.

Proof of Proposition 2. Notice that if $X \leq G$, then $\tilde{X}$ is a maximal intersection in $G$, and if $X$ is itself a maximal intersection, then $\tilde{X}=X$. So, by Lemma 9 , the map sending the equivalence class containing $X$ to $\tilde{X}$ induces a bijection from the set of the equivalence classes to the set of the maximal intersections in $G$. Moreover, if $X_{1}, X_{2} \in \mathcal{M}(G)$, then $X_{1} \leq X_{2}$ if and only if $\mathcal{N}_{G}\left(X_{1}\right) \subseteq \mathcal{N}_{G}\left(X_{2}\right)$.

We conclude this section with an example showing that if $X_{1}, X_{2} \in \mathcal{M}(G)$, then it is not necessarily true that $\left\langle X_{1}, X_{2}\right\rangle \in \mathcal{M}(G)$. Let $\mathbb{F}$ be the field with three elements, and let $C=\langle-1\rangle$ be the multiplicative group of $\mathbb{F}$. Let $V=\mathbb{F}^{3}$ be a 3 -dimensional vector space over $\mathbb{F}$ and let $\sigma=(1,2,3) \in \operatorname{Sym}(3)$. The wreath product $H=C \imath\langle\sigma\rangle$ has an irreducible action on $V$ defined as follows: if $v=\left(f_{1}, f_{2}, f_{3}\right) \in V$ and $h=\left(c_{1}, c_{2}, c_{3}\right) \sigma^{i} \in H$, then $v^{h}=\left(f_{1 \sigma^{-i}} c_{1 \sigma^{-i}}, f_{2 \sigma^{-i}} c_{2 \sigma^{-i}}, f_{3 \sigma^{-i}} c_{3 \sigma^{-i}}\right)$. Consider the semidirect product $G=V \rtimes H$ and let $v=(1,-1,0) \in V$. Since $H$ and $H^{v}$ are two maximal subgroups of $G, K:=H \cap H^{v}=$ $C_{H}(v)=\{(1,1, z) \mid z \in C\} \cong C_{2}$ is a maximal intersection in $G$. Since $G / V \cong H$ and $\operatorname{Frat}(H)=1, V$ is also a maximal intersection in $G$. However, $V K$ is not a maximal intersection in $G$. Indeed, if $X$ is a maximal intersection in $G$ containing $V$, then $X=V Y$ with $Y$ a maximal intersection in $H$. But $H \cong C_{2} \times \operatorname{Alt}(4)$ and the unique subgroup of order 2 of $H$ that can be obtained as an intersection of maximal subgroups is $\{(z, z, z) \mid z \in C\}$.

The following elementary remark is used several times throughout the paper. 
LEMma 10. If a finite Frattini-free nilpotent group $X$ contains $t$ maximal subgroups that intersect trivially, then $|X|$ is a product of at most $t$ (not necessarily distinct) primes.

3. Proof of Theorem 4. Recall that the Möbius function $\mu_{G}$ is defined on the subgroup lattice of $G$ as $\mu_{G}(G)=1$ and $\mu_{G}(H)=-\sum_{H<K} \mu_{G}(K)$ for any $H<G$. If $H \leq G$ cannot be expressed as an intersection of maximal subgroups of $G$, then $\mu_{G}(H)=0$ (see [12, Theorem 2.3]), so for every $H \in \mathcal{M}(G)$, the value $\mu_{G}(H)$ can be completely determined from the knowledge of the lattice $\mathcal{M}(G)$. The following result could be easily deduced from [15, Theorem 2.6]. We prefer to give a direct proof.

Proposition 11. Let $G$ be a finite soluble group. For every irreducible $G$-module $V$ define $q(V)=\left|\operatorname{End}_{G}(V)\right|$, set $\theta(V)=0$ if $V$ is a trivial $G$-module, and $\theta(V)=1$ otherwise, and let $\delta(V)$ be the number of chief factors $G$-isomorphic to $V$ and complemented in an arbitrary chief series of $G$. Let $\mathcal{V}(G)$ be the set of irreducible $G$-modules $V$ with $\delta(V) \neq 0$. Then

$$
\mu_{G}(1)= \begin{cases}\prod_{V \in \mathcal{V}(G)}(-1)^{\delta(V)}|V|^{\theta(V) \delta(V)} q(V){ }^{\left(\begin{array}{c}
\delta(V) \\
2
\end{array}\right)} & \text { if } \prod_{V \in \mathcal{V}(G)}|V|^{\delta(V)}=|G|, \\
0 & \text { otherwise. }\end{cases}
$$

Proof. We prove the statement by induction on the order of $G$. Let $N$ be a minimal normal subgroup of $G$. By [13, Lemma 3.1]

$$
\mu_{G}(1)=\mu_{G / N}(1) \sum_{K \in \mathcal{K}} \mu_{G}(K),
$$

denoting by $\mathcal{K}$ the set of all subgroups of $G$ which complement $N$. If $\mathcal{K}=\varnothing$, then $N$ is a non-complemented chief factor of $G$ and $\mu_{G}(1)=0$. Moreover in this case, $\prod_{V \in \mathcal{V}(G)}|V|^{\delta(V)} \leq|G| /|N|<|G|$. In any case, since $N$ is a minimal normal subgroup of $G$ and $G$ is soluble, if $K \in \mathcal{K}$, then $K$ is a maximal subgroup of $G$ and consequently $\mu_{G}(K)=-1$. Thus, $\mu_{G}(1)=-\mu_{G / N}(1) \cdot c$, where $c$ is the number of complements of $N$ in $G$. To conclude it suffices to notice that, by [10, Satz 3], $c=|N|^{\theta(N)} q(N)^{\delta(N)-1}$.

COROLlaRY 12. If $X \cong C_{p_{1}}^{m_{1}} \times \cdots \times C_{p_{t}}^{m_{t}}$, then $\mu_{X}(1)=(-1)^{m_{1}} p_{1}^{\left(\begin{array}{c}m_{1} \\ 2\end{array}\right)} \cdots(-1)^{m_{t}} p_{t}^{\left(\begin{array}{c}m_{t} \\ 2\end{array}\right)}$.

LEMma 13. Let $G$ be a finite group and assume $G \in \mathfrak{M}$. If $N$ is a normal subgroup of $G$ containing $\operatorname{Frat}(G)$, then

(1) $\mu_{G}(N) \neq 0$;

(2) $N$ is a maximal-intersection in $G$;

(3) $\operatorname{Frat}(G / N)=1$;

(4) $G / N \in \mathfrak{M}$.

Proof. Since $G \in \mathfrak{M}$, there exists a finite nilpotent group with $\mathcal{M}(G) \cong \mathcal{M}(X)$. We have $\mathcal{M}(G / \operatorname{Frat}(G)) \cong \mathcal{M}(G) \cong \mathcal{M}(X) \cong \mathcal{M}(X / \operatorname{Frat}(X))$, and this implies $\mu_{X / \operatorname{Frat}(X)}(1)=\mu_{G / \operatorname{Frat}(G)}(1)$. By Corollary $12, \quad \mu_{X / \operatorname{Frat}(X)}(1) \neq 0$ and therefore $\mu_{G / \operatorname{Frat}(G)}(1) \neq 0$. If $N$ is a normal subgroup of $G$ containing $\operatorname{Frat}(G)$, then we deduce from [13, Lemma 3.1] that $\mu_{G}(N)=\mu_{G / N}(1)$ divides $\mu_{G / \operatorname{Frat}(G)}(1)$. As a consequence, $\mu_{G}(N) \neq 0$ and $N$ is a maximal intersection in $G$. This implies in particular $\operatorname{Frat}(G / N)=1$. Finally, there exists $Y \leq X$ such that $\mathcal{M}(G / N) \cong \mathcal{M}(X / Y)$, so $G / N \in \mathfrak{M}$. 
LEMMA 14. Let $H$ be a finite supersoluble group and $V$ a faithful irreducible $H$-module. Consider the semidirect product $G=V \rtimes H$. Suppose that there exists a finite nilpotent group $X$ with $\mathcal{M}(G) \cong \mathcal{M}(X)$. Then $V$ is cyclic of prime order.

Proof. Since $\mathcal{M}(X) \cong \mathcal{M}(X / \operatorname{Frat}(X))$, we may assume $\operatorname{Frat}(X)=1$. There exist $v$ and $w$ in $V$ such that $C_{H}(v) \cap C_{H}(w)=1$ (see [19, Theorem A]). This implies that $H, H^{v}, H^{w}$ are maximal subgroups of $G$ with trivial intersection. But then also $X$ must contain three maximal subgroups with trivial intersection, and consequently, by Lemma 10, $|X|$ is the product of at most three (not necessarily distinct) primes. Suppose $|V|=p^{a}$, with $p$ a prime and $a \geq 2$. Since $\operatorname{Frat}(X)=1$, it follows from Corollary 12 that $\mu_{X}(1) \neq 0$. Moreover, by Proposition 11, $\mu_{X}(1)=\mu_{G}(1)$ is divisible by $p^{a}$. By Corollary 12, this is possible only if $X \cong C_{p} \times C_{p} \times C_{p}$ and $\mu_{X}(1)=\mu_{G}(1)=-p^{3}$. By Proposition 11, $|V|$ divides $\mu_{G}(1)$ so $V$ is a $p$-group. By Lemma $13, V \in \mathcal{M}(G)$. Since $V$ is a minimal element in $\mathcal{M}(G)$, it follows that $\mathcal{M}(H) \cong \mathcal{M}(G / V) \cong \mathcal{M}\left(C_{p} \times C_{p}\right)$ and therefore, by Corollary $12, \mu_{H}(1)=p$. Moreover 2 is the maximal length of a chain in $\mathcal{M}(H)$ and $\operatorname{Frat}(H)=1$ by Lemma 13. So $H$ is a supersoluble group in which the intersection of any pair of maximal subgroups is trivial. This implies that $|H|$ is the product of two primes, say $p_{1}$ and $p_{2}$, and we may assume that $H$ has a normal subgroup of order $p_{1}$. By Proposition 11 , $\mu_{H}(1)=1$ if $H$ is cyclic, $\mu_{H}(1)=p_{1}$ otherwise. Since $\mu_{H}(1)=p$, it follows that $O_{p}(H) \neq 1$, in contradiction with the fact that $V$ is a faithful irreducible $H$-module of p-power order.

LEMMA 15. If $G$ is a finite almost simple group, then there exist maximal subgroups $M_{1}, \ldots, M_{t}$ of $G$, with $t \leq 5$, with the property that $M_{1} \cap \cdots \cap M_{t}=1$.

Proof. The result follows from [5, Theorem 1], except when $S=\operatorname{soc}(G)$ is an alternating group or a classical group and all the primitive actions of $G$ are of standard type. If $\operatorname{soc}(G)$ is of alternating type, then the result follows from [7, Corollaries 1.4, 1.5, Remark 1.6] (see also [9, Lemma 2] and its proof). In the case of classical groups, we are done if we are able to build up a non-standard action by taking primitive actions with stabilizer in one of the Aschbacher classes $\mathscr{C}_{2}, \mathscr{C}_{3}, \mathscr{C}_{4}, \mathscr{C}_{5}, \mathscr{C}_{6}, \mathscr{C}_{7}$. For this purpose, we use [14, Tables 3.5.A. 3.5.B, 3.5.C, 3.5.D, 3.5.E and 3.5.F] (and the similar tables in [4] if the dimension of $G$ is up to 12). We need to be careful because a subgroup $H$ in one of the given Aschbacher classes of $G$ may not actually be maximal in $G$. As it is explained in [14, Section 3.4], to avoid this possibility, we need to select $H$ in such a way that when we look to the corresponding row in the table, we do not find restrictive conditions in column VI and the homomorphism $\pi$ described in column $\mathrm{V}$ is the identity. A subgroup with these properties can be found, except in the following three cases:

(1) $S=\Omega_{2 p}^{+}$(2) and $p$ is an odd prime (and we may assume $p \geq 5$, since $\Omega_{6}^{+}(2) \cong \operatorname{Alt}(8)$ ). In this case, $|G: S| \leq 2$. Let $V$ be the natural module for $G$, and let $\Omega$ be the set of nondegenerate plus-type subspaces of dimension $p+1$. Then $G$ acts primitively on this set, and by the proof of [6, Theorem 6.13], it contains three maximal subgroups $M_{1}, M_{2}, M_{3}$ such that $M_{1} \cap M_{2} \cap M_{3} \cap S=1$, so $t \leq 4$.

(2) $S=\mathrm{P} \Omega_{2 p}^{+}(5)$ and $p$ is an odd prime. Again, let $V$ be the natural module for $S$, and let $\Omega$ be the set of nondegenerate plus-type subspaces of dimension $p+1$. Then $G$ acts primitively on this set. Arguing as in the proof of [6, Theorem 6.13], three subspaces in $\Omega$ can be exhibited with the property that if $g \in \mathrm{O}_{2 p}^{+}(5)$ stabilizes each of them, then, with respect to a suitable basis, $g$ is represented either by a scalar matrix or by the matrix 


$$
\pm\left(\begin{array}{ccc}
I_{2 p-2} & 0 & 0 \\
0 & 0 & 1 \\
0 & 1 & 0
\end{array}\right)
$$

Let $M_{1}, M_{2}, M_{3}$ be the stabilizers in $G$ of these subspaces. We have $\mid M_{1} \cap M_{2} \cap M_{3} \cap$ $\mathrm{PO}_{2 p}^{+}(5) \mid \leq 2$, so $\left|M_{1} \cap M_{2} \cap M_{3} \cap G\right| \leq 4$ and consequently there exist $M_{4}$ and $M_{5}$ such that $M_{1} \cap M_{2} \cap M_{3} \cap M_{4} \cap M_{5}=1$.

(3) $S=\Omega_{p}(q)$ with $p \geq 7$ a prime, $q=q_{0}^{t}$ with $q_{0}$ an odd prime, and $t$ a power of 2 . In this case, let $V$ be the natural module for $S$ and $\Omega$ the set of the $2 m$-dimensional nondegenerate subspaces of $V$ of plus-type if $p=4 m+1$, or the set of the $(2 m+1)$ dimensional nondegenerate subspaces $X$ of $V$ with the property that $X^{\perp}$ has plus type if $p=4 m+3$. Then, $G$ acts primitively on $\Omega$, and by [6, Theorem 6.11], the restriction of this action to $S$ has a base of size 2. By [11, Theorem 1.2], each element of $G$ has a regular cycle. Since $G / S$ is metacyclic, it follows that the action of $G$ on $\Omega$ has a base of size at most 4 . As a consequence, we can find four point stabilizers with trivial intersection.

LEMMA 16. If $G$ is a finite monolithic primitive group with non-abelian socle, then there is no finite nilpotent group $X$ with $\mathcal{M}(G) \cong \mathcal{M}(X)$.

Proof. Assume, by contradiction, that there exists a finite nilpotent group $X$ with $\mathcal{M}(X) \cong \mathcal{M}(G)$. Since $\mathcal{M}(X) \cong \mathcal{M}(X / \operatorname{Frat}(X))$, we may assume $\operatorname{Frat}(X)=1$. There exists a finite nonabelian simple group $S$ such that $N=\operatorname{soc}(G)=S_{1} \times \ldots \times S_{n}$, with $S_{i} \cong S$ for $1 \leq i \leq n$.

Suppose first that $n \geq 2$. Let $\psi$ be the map from $N_{G}\left(S_{1}\right)$ to $\operatorname{Aut}(S)$ induced by the conjugacy action on $S_{1}$. Set $H=\psi\left(N_{G}\left(S_{1}\right)\right)$, and note that $H$ is an almost simple group with socle $S=\operatorname{Inn}(S)=\psi\left(S_{1}\right)$. Let $T:=\left\{t_{1}, \ldots, t_{n}\right\}$ be a right transversal of $N_{G}\left(S_{1}\right)$ in $G$; the map

$$
\phi_{T}: G \rightarrow H z \operatorname{Sym}(n)
$$

given by

$$
g \mapsto\left(\psi\left(t_{1} g t_{1 \pi_{g}}^{-1}\right), \ldots, \psi\left(t_{n} g t_{n \pi_{g}}^{-1}\right)\right) \pi_{g},
$$

where $\pi_{g} \in \operatorname{Sym}(n)$ satisfies $t_{i} g t_{i \pi_{g}}^{-1} \in N_{G}\left(S_{1}\right)$ for all $1 \leq i \leq n$, is an injective homomorphism. So we may identify $G$ with its image in $H$ 2 $\operatorname{Sym}(n)$; in this identification, $N$ is contained in the base subgroup $H^{n}$ and $S_{i}$ is a subgroup of the $i$ th component of $H^{n}$. By Lemma 13, Frat $(G / N)=1$ and so there exist $u$ maximal subgroups $M_{1}, \ldots, M_{u}$ of $G$ such that

$$
N=M_{1} \cap \cdots \cap M_{u}<M_{1} \cap \cdots \cap M_{u-1}<\cdots<M_{1} \cap M_{2}<M_{1}<G .
$$

Let $R$ be a maximal subgroup of $H$ with $H=R S$ and set $K=R \cap S$. We must have $K \neq 1$ (see, for example, the last paragraph of the proof of the main theorem in [16]). Notice that $L:=G \cap(R 2 \operatorname{Sym}(n))$ is a maximal subgroup of $G([2]$ Proposition 1.1.44). We have $D:=L \cap M_{1} \cap \cdots \cap M_{u}=L \cap N=K^{n}$. Choose a subset $\left\{s_{1}, \ldots, s_{m}\right\}$ of $S$ with minimal cardinality with respect to the property $K \cap K^{s_{1}} \cap \cdots \cap K^{s_{m}}=1$. Set 


$$
\begin{aligned}
& \alpha_{1}=\left(s_{1}, \ldots, s_{1}\right), \alpha_{2}=\left(s_{2}, \ldots, s_{2}\right), \ldots, \alpha_{m}=\left(s_{m}, \ldots, s_{m}\right), \\
& \beta_{1}=\left(s_{1}, 1, \ldots, 1\right), \beta_{2}=\left(s_{2}, 1, \ldots, 1\right), \ldots, \beta_{m}=\left(s_{m}, 1, \ldots, 1\right), \\
& \gamma_{1}=\left(1, s_{1}, \ldots, s_{1}\right), \gamma_{2}=\left(1, s_{2}, \ldots, s_{2}\right), \ldots, \gamma_{m}=\left(1, s_{m}, \ldots, s_{m}\right) .
\end{aligned}
$$

For $1 \leq i \leq m$, set

$$
\begin{aligned}
& A_{i}:=L^{\alpha_{i}} \cap \cdots \cap L^{\alpha_{m}} \cap D, \\
& B_{i}:=L^{\beta_{i}} \cap \cdots \cap L^{\beta_{m}} \cap L^{\gamma_{1}} \cap \cdots \cap L^{\gamma_{m}} \cap D, \\
& C_{i}:=L^{\gamma_{i}} \cap \cdots \cap L^{\gamma_{m}} \cap D .
\end{aligned}
$$

We have

$$
1=A_{1}<\cdots<A_{m}<D, \quad 1=B_{1}<\cdots<B_{m}<C_{1}<\cdots<C_{m}<D .
$$

In particular,

$$
\left\{M_{1}, \ldots, M_{t}, L, L^{\alpha_{1}}, \ldots, L^{\alpha_{m}}\right\}, \quad\left\{M_{1}, \ldots, M_{t}, L, L^{\beta_{1}}, \ldots, L^{\beta_{m}}, L^{\gamma_{1}}, \ldots, L^{\gamma_{m}}\right\}
$$

are two families of maximal subgroups of $G$ that are minimal with respect to the property that their intersection is the trivial subgroup. However, the assumption $\mathcal{M}(G) \cong \mathcal{M}(X)$ implies that all the families of maximal subgroups of $G$ with this property must have the same size.

We may therefore assume that $G$ is a finite almost simple group. Since $\operatorname{Frat}(X)=1$, by Corollary $12,0 \neq \mu_{X}(1)=\mu_{G}(1)$. By Lemma $15, G$ contains $t \leq 5$ maximal subgroups with trivial intersection. But then $X$ satisfies the same properties, and consequently, by Lemma $10,|X|$ is the product of at most $t \leq 5$ primes. It follows from Corollary 12 that $\mu_{X}(1)=\mu_{G}(1)$ is divisible by at most two different primes. By [13, Theorem 4.5], $|G|$ divides $m \cdot \mu_{G}(1)$, where $m$ is the square-free part of $\left|G / G^{\prime}\right|$. So, if $S=\operatorname{soc}(G)$, then, since $S \leq G^{\prime}, m$ divides $|G / S|$ and consequently $|S|$ divides $\mu_{G}(1)=\mu_{X}(1)$. But then $|S|$ is divisible by at most two different primes, so it is soluble by Burnside's $p^{a} q^{b}$-theorem, a contradiction.

Proof of Theorem 4. We prove our statement by induction on the order of $G$. If $\operatorname{Frat}(G) \neq 1$, then $\mathcal{M}(G / \operatorname{Frat}(G)) \cong \mathcal{M}(X / \operatorname{Frat}(X))$, so $G / \operatorname{Frat}(G)$ is supersoluble by induction. But this implies that $G$ itself is supersoluble. So we may assume $\operatorname{Frat}(G)=1$. Assume, by contradiction, that $G$ is not soluble. Then, there exists a non-abelian chief factor $R / S$ of $G$. Let $L=G / C_{G}(R / S)$. Notice that $L$ is a primitive monolithic group whose socle is isomorphic to $R / S$. By Lemma $13, C_{G}(R / S)$ is a maximal intersection in $G$. But then $\mathcal{M}(L) \cong \mathcal{M}(X / Y)$ for a suitable normal subgroup $Y$ of $X$, in contradiction with Lemma 16. So we may assume that $G$ is soluble. Assume by contradiction that $G$ is not supersoluble. Let $1=N_{0}<N_{1}<\cdots<N_{u}=G$ be a chief series of $G$, and let $j$ be the largest positive integer with the property that the chief factor $N_{j} / N_{j-1}$ is not cyclic. Let $V=N_{j} / N_{j-1}$ and $H=G / C_{G}(V)$. By Lemma 13 and Proposition $11, N_{j} / N_{j-1}$ is a complemented chief factor of $G$. Let $K / N_{j-1}$ be a complement of $N_{j} / N_{j-1}$ in $G / N_{j-1}$ and set $M=N_{j-1} C_{K}(V)$. It turns out that $G / M \cong V \rtimes H$. Again by Lemma $13, M$ is a maximal intersection in $G$, so there exists $Y \leq X$ such that $\mathcal{M}(G / M) \cong \mathcal{M}(X / Y)$. By our choice of the index $j$, the factor group $G / N_{j}$ is supersoluble. Since $N_{j} \leq C_{G}(V)$, also $H$ is supersoluble. But then it follows from Lemma 14 that $V$ is cyclic of prime order, in contradiction with our assumption. 


\section{Frattini-free groups in $\mathfrak{D}$ and $\mathfrak{M}$.}

Proof of Proposition 6. Assume that $X$ is a finite nilpotent group with $\Delta(X) \cong \Delta(G)$. Since $\operatorname{Frat}(G)=1$, the unique isolated vertex in $\Delta(G)$ is the one corresponding to the identity subgroup. The same must be true in $\Delta(X)$ and therefore $\operatorname{Frat}(X)=1$. Hence, $X$ is a direct product of elementary abelian groups. In particular, every subgroup of $X$ is a maximal intersection in $X$, so the lattice $\mathcal{M}(X)$ coincides with the entire subgroup lattice $\mathcal{L}(X)$ of $X$. This is equivalent to say that if $Y_{1}$ and $Y_{2}$ are different subgroups of $G$, then $\mathcal{N}_{G}\left(Y_{1}\right) \neq \mathcal{N}_{G}\left(Y_{2}\right)$. Again, the same property holds for $\Delta(G)$ and consequently $\mathcal{M}(G) \cong \mathcal{L}(G)$. So by Proposition $2, \mathcal{L}(G) \cong \mathcal{L}(X)$, and the conclusion follows from [17, Theorem 2.5.10].

Lemma 17. Suppose that $X_{1}$ and $X_{2}$ are finite groups. If no simple group is a homomorphic image of both $X_{1}$ and $X_{2}$ then $\mathcal{M}\left(X_{1} \times X_{2}\right) \cong \mathcal{M}\left(X_{1}\right) \times \mathcal{M}\left(X_{2}\right)$.

Proof. A maximal subgroup $M$ of a direct product $X_{1} \times X_{2}$ is of standard type if either $M=Y_{1} \times X_{2}$ with $Y_{1}$ a maximal subgroup of $X_{1}$ or $M=X_{1} \times Y_{2}$ with $Y_{2}$ a maximal subgroup of $X_{2}$. A maximal subgroup $M$ of $X_{1} \times X_{2}$ is of diagonal type if there exist a maximal normal subgroup $N_{1}$ of $X_{1}$, a maximal normal subgroup $N_{2}$ of $X_{2}$, and an isomorphism $\phi: X_{1} / N_{1} \rightarrow X_{2} / N_{2}$ such that $M=\left\{\left(x_{1}, x_{2}\right) \in H_{1} \times H_{2} \mid \phi\left(x_{1} N_{1}\right)=x_{2} N_{2}\right\}$. By [18, Chapter 2, (4.19)], a maximal subgroup of $X_{1} \times X_{2}$ is either of standard type or of diagonal type. If no simple group is a homomorphic image of both $X_{1}$ and $X_{2}$, then all the maximal subgroups of $X_{1} \times X_{2}$ are of standard type. In particular, $K \in \mathcal{M}\left(X_{1} \times X_{2}\right)$ if and only if $K=K_{1} \times K_{2}$, with $K_{1} \in \mathcal{M}\left(X_{1}\right)$ and $K_{2} \in \mathcal{M}\left(X_{2}\right)$.

LEMMA 18. The following hold:

(1) If $G=H_{1} \times \cdots \times H_{t-1} \in \Lambda\left(p_{1}, \ldots, p_{t}\right)$, with $H_{i} \cong C_{p_{i}}^{n_{i}} \rtimes C_{p_{i}+1}$, then $\mathcal{M}(G) \cong$ $\mathcal{M}\left(C_{p_{1}}^{n_{1}+1} \times \cdots \times C_{p_{t-1}}^{n_{t-1}+1}\right)$.

(2) If $G=H_{1} \times \cdots \times H_{t-1} \times C_{p_{1}} \in \Lambda^{*}\left(p_{1}, \ldots, p_{t}\right)$ with $H_{i} \cong C_{p_{i}}^{n_{i}} \rtimes C_{p_{i}+1}$, then $\mathcal{M}(G) \cong \mathcal{M}\left(C_{p_{1}}^{n_{1}+1} \times \cdots \times C_{p_{t-1}}^{n_{t-1}+1} \times C_{p_{t}}\right)$.

Proof. Let $H \cong C_{p}^{n} \rtimes C_{q}$ be a $P$-group. By [17, Theorem 2.2.3], the subgroup lattices of $H$ and $C_{p}^{n+1}$ are isomorphic, and consequently, $\mathcal{M}(H) \cong \mathcal{M}\left(C_{p}^{n+1}\right)$. Now assume $G=H_{1} \times \cdots \times H_{t-1} \in \Lambda\left(p_{1}, \ldots, p_{t}\right)$, with $H_{i} \cong C_{p_{i}}^{n_{i}} \rtimes C_{p_{i}+1}$. By Lemma 17,

$$
\begin{aligned}
\mathcal{M}(G) & \cong \mathcal{M}\left(H_{1} \times \cdots \times H_{t-1}\right) \cong \mathcal{M}\left(H_{1}\right) \times \cdots \times \mathcal{M}\left(H_{t-1}\right) \\
& \cong \mathcal{M}\left(C_{p_{1}}^{n_{1}+1}\right) \times \cdots \times \mathcal{M}\left(C_{p_{t-1}}^{n_{t-1}+1}\right) \cong \mathcal{M}\left(C_{p_{1}}^{n_{1}+1} \times \cdots \times C_{p_{t-1}}^{n_{t-1}+1}\right) .
\end{aligned}
$$

This proves (1). If $G=H_{1} \times \cdots \times H_{t-1} \times C_{p_{1}} \in \Lambda^{*}\left(p_{1}, \ldots, p_{t}\right)$ with $H_{i} \cong C_{p_{i}}^{n_{i}} \rtimes C_{p_{i}+1}$, then, again by Lemma 17 ,

$$
\begin{aligned}
\mathcal{M}(G) & \cong \mathcal{M}\left(H_{1} \times \cdots \times H_{t-1} \times C_{p_{1}}\right) \\
& \cong \mathcal{M}\left(H_{1}\right) \times \cdots \times \mathcal{M}\left(H_{t-1}\right) \times \mathcal{M}\left(C_{p_{1}}\right) \\
& \cong \mathcal{M}\left(C_{p_{1}}^{n_{1}+1}\right) \times \cdots \times \mathcal{M}\left(C_{p_{t-1}}^{n_{t-1}+1}\right) \times \mathcal{M}\left(C_{p_{1}}\right) \\
& \cong \mathcal{M}\left(C_{p_{1}}^{n_{1}+1}\right) \times \cdots \times \mathcal{M}\left(C_{p_{t-1}}^{n_{t-1}+1}\right) \times \mathcal{M}\left(C_{p_{t}}\right) \\
& \cong \mathcal{M}\left(C_{p_{1}}^{n_{1}+1} \times \cdots \times C_{p_{t-1}}^{n_{t-1}+1} \times C_{p_{t}}\right) .
\end{aligned}
$$

So (2) is also proved. 
Proof of Proposition 7. First, we prove by induction on the order of $G$ that if $G \in \mathfrak{M}$, then $G$ is as described in the statement. Let $M$ be a normal subgroup of $G$. By Lemma 13, $\operatorname{Frat}(G / M)=1$ and $G / M \in \mathfrak{M}$. Hence, $G / M$ satisfies the same assumptions as $G$. During the proof, we will use several times, without an explicit mention, this remark.

Let $N$ be a minimal normal subgroup of $G$. By Theorem 4 , there exists a prime $p$ such that $N \cong C_{p}$. Moreover, since $\operatorname{Frat}(G)=1, N$ has a complement, say $K$ in $G$. Since $K \cong G / N$, by induction $K=H_{1} \times \cdots \times H_{u}$, where $H_{1}, \ldots, H_{u}$ have coprime orders and are as described in the statement.

First assume that $N$ is central in $G$. If $p$ does not divide the order of $K$, then $G=H_{1} \times \cdots \times H_{u} \times N$ is a factorization with the required properties. Otherwise, there exists a unique $i$ such that $p$ divides $\left|H_{i}\right|$. It is not restrictive to assume $i=u$. If $H_{u}$ is either elementary abelian or $H_{u} \in \Lambda\left(p_{1}, \ldots, p_{t}\right)$ with $p_{1}=p$, then we set $\tilde{H}_{u}=H_{u} \times N \cong$ $H_{u} \times C_{p}$ and the factorization $G=H_{1} \times \cdots \times H_{u-1} \times \tilde{H}_{u}$ satisfies the required properties. In the other cases, there exist a prime $q \neq p$ and a normal subgroup $L$ of $H_{u}$ such that $J=H_{u} / L$ is isomorphic either to $C_{q} \rtimes C_{p}$ or to $\left(C_{p} \rtimes C_{q}\right) \times C_{p}$. Since $T=N \times J \cong$ $G /\left(H_{1} \times \cdots \times H_{u-1} \times L\right) \in \mathfrak{M}$, there exists a Frattini-free nilpotent group $X$ with $\mathcal{M}(X) \cong \mathcal{M}(T)$. Notice that since $\operatorname{Frat}(X)=1, X$ is a direct product of elementary abelian groups, so we may apply Corollary 12 when it is needed. If $J \cong C_{q} \rtimes C_{p}$, then $\mu_{X}(1)=$ $\mu_{T}(1)=-p \cdot q$ and $|X|$ is the product of three primes, but this possibility is excluded by Corollary 12 . If $J \cong\left(C_{p} \rtimes C_{q}\right) \times C_{p}$, then $\mu_{X}(1)=\mu_{T}(1)=p^{2}$, again in contradiction with Corollary 12.

Now assume that $N$ is not central. Notice that $G / C_{G}(N)$, being isomorphic to a subgroup of $\operatorname{Aut}(N)$, is cyclic. Since $\operatorname{Frat}\left(G / C_{G}(N)\right)=1$, we deduce $G / C_{G}(N) \cong C_{q}$, where $q$ is a square-free positive integer. Moreover, there exists a Frattini-free nilpotent group $X$ such that $\mathcal{M}(X) \cong \mathcal{M}\left(G / C_{K}(N)\right)$. Since $G / C_{K}(N) \cong C_{p} \rtimes C_{q}$, the identity subgroup of $G / C_{K}(N)$ can be obtained as the intersection of two conjugated subgroups of order $q$. By Lemma 10, $|X|$ is the product of two primes, and consequently, $\mathcal{M}\left(G / C_{K}(N)\right) \cong \mathcal{M}(X)$ cannot contain chains of length $>2$. But then $q$ is a prime. In particular, there exists a unique $i$ such that $q$ divides $\left|H_{i}\right|$. It is not restrictive to assume $i=u$. Notice that $C_{q} \cong H_{u} / C_{H_{u}}(N)$, so $q$ divides $\left|H_{u} / H_{u}^{\prime}\right|$. We distinguish the different possibilities for $H_{u}$ and determine the structure of $\mathrm{NH}_{u}$ in each case.

First assume $H_{u}=C_{q}^{t}$, for some $t \in \mathbb{N}$. Then, $G /\left(H_{1} \times \cdots \times H_{u-1}\right) \cong N H_{u} \cong\left(C_{p} \rtimes\right.$ $\left.C_{q}\right) \times C_{q}^{t-1}$. If $t \geq 2$, then $Y_{1}=\left(C_{p} \rtimes C_{q}\right) \times C_{q}$ would be an epimorphic image of $G$. Consequently, by Lemma 10, there would exist a nilpotent group $X$ whose order is the product of three primes such that $\mu_{X}(1)=\mu_{Y_{1}}(1)=-p \cdot q$, in contradiction with Corollary 12 . Thus, $t=1$, and consequently, $N H_{u} \in \Lambda(p, q)$.

Assume $H_{u}=T_{1} \times \cdots \times T_{t-1} \in \Lambda\left(p_{1}, \ldots, p_{t}\right)$, with $T_{j} \cong C_{p_{j}}^{n_{j}} \rtimes C_{p_{j+1}}$. Since $H_{u}$ is a direct product of non-abelian $P$-groups, $\left|H_{u} / H_{u}^{\prime}\right|$ is not divisible by $p_{1}$. On the other hand, $q$ divides $\left|H_{u} / H_{u}^{\prime}\right|$, hence $q \neq p_{1}$ and there exists $1 \leq i \leq t-1$ such that $q=p_{i+1}$. Moreover, since $H_{u} / C_{H_{u}}(N) \cong C_{q}$, it follows that $C_{H_{u}}(N)=\left(\prod_{j \neq i} T_{j}\right) \times C_{p_{i}}^{n_{i}}$. Let $r=p_{i}$ and $R$ a (noncentral) normal subgroup of $T_{i}$ with order $r$. A Sylow $q$-subgroup $Q$ of $T_{i}$ centralizes neither $N$ nor $R$. The semidirect product $Y_{2}=(N \times R) \rtimes Q \cong\left(C_{p} \times C_{r}\right) \rtimes C_{q}$ is an epimorphic image of $G$, and consequently, there exists a nilpotent group $X$ whose order is the product of three primes (by Lemma 10) such that $\mu_{X}(1)=\mu_{Y_{2}}(1)$ is divisible by $p \cdot r$. By Corollary 12 and Proposition 11, this is possible only if $p=r, X \cong C_{p}^{3}, \mu_{X}(1)=-p^{3}$ and $N$ and $R$ are $Q$-isomorphic (and consequently $G$-isomorphic). But then $N T_{i} \cong C_{p}^{1+n_{i}} \rtimes C_{q}$ is a $P$-group and $N H_{u}=T_{1} \times \cdots \times T_{i-1} \times N T_{i} \times T_{i+1} \times \cdots \times T_{t-1} \in \Lambda\left(p_{1}, \ldots, p_{t}\right)$. 
Assume $H_{u}=T_{1} \times \cdots \times T_{t-1} \times L \in \Lambda^{*}\left(p_{1}, \ldots, p_{t}\right)$, with $T_{j} \cong C_{p_{j}}^{n_{j}} \rtimes C_{p_{j+1}}$ and $L$ a group of order $p_{1}$. If $q \neq p_{1}$, then $q=p_{i+1}$ for some $1 \leq i \leq t$, and we may repeat the previous argument to deduce that $N T_{i}$ is a $P$-group and $N H_{u}=T_{1} \times \cdots \times T_{i-1} \times N T_{i} \times$ $T_{i+1} \times \cdots \times T_{t-1} \times L \in \Lambda^{*}\left(p_{1}, \ldots, p_{t}\right)$. If $q=p_{1}$, then $N L$ is a $P$-group of order $p \cdot p_{1}$ and $N H_{u}=N L \times T_{1} \times \cdots \times T_{t-1} \in \Lambda\left(p, p_{1}, \ldots, p_{t}\right)$.

We conclude that in any case one of the following occurs:

(1) $N H_{u} \in \Lambda\left(p, p_{1}, \ldots, p_{t}\right)$,

(2) $N H_{u} \in \Lambda\left(p_{1}, \ldots, p_{t}\right)$,

(3) $N H_{u} \in \Lambda^{*}\left(p_{1}, \ldots, p_{t}\right)$.

If $p$ does not divide $\left|H_{1}\right| \cdots\left|H_{u-1}\right|$, then the factorization $H_{1} \times \ldots H_{u-1} \times N H_{u}$ satisfies the requirements of the statement. Otherwise, we may assume that $p$ divides $\left|H_{1}\right|$. Notice that in this case $p$ does not divide $H_{u}$, so $N H_{u} \in \Lambda\left(p, p_{1}, \ldots, p_{t}\right)$. If $H_{1}$ admits a non-central chief factor of order $p$, then there exists a prime $r$ such that $Y_{3}=\left(C_{p} \rtimes C_{q}\right) \times\left(C_{p} \rtimes C_{r}\right)$ is an epimorphic image of $G$. There would exist a nilpotent group $X$ with $\mu_{X}(1)=\mu_{Y_{3}}(1)$. However by Proposition $11, \mu_{Y_{3}}(1)=p^{2} \cdot q^{\eta}$, with $\eta=1$ if $q=r, \eta=0$ otherwise, while by Corollary $12, p$ cannot divide $\mu_{X}(1)$ with multiplicity equal to 2 . The only possibility that remains is $H_{1} \cong C_{p}^{t}$. If $t \geq 2$, then $Y_{4}=\left(C_{p} \rtimes C_{q}\right) \times C_{p}^{2}$ is an epimorphic image of $G$, and there would exist a nilpotent group $X$ with $\mu_{X}(1)=\mu_{Y_{4}}(1)=p^{2}$, again in contradiction with Corollary 12. So $t=1$ and $H_{1} \times N H_{u} \in \Lambda^{*}\left(p, p_{1}, p_{2}, \ldots, p_{t}\right)$. Setting $\tilde{H}_{1}=$ $H_{1} \times N H_{u}$, we conclude that $\tilde{H}_{1} \times H_{2} \times \cdots \times H_{u-1}$ is the factorization we are looking for.

Conversely, assume that $G=H_{1} \times \cdots \times H_{u}$ is a factorization with the properties described by the statement. By Lemma 18 , for every $1 \leq i \leq u$, there exists a nilpotent group $X_{i}$ such that $\mathcal{M}\left(H_{i}\right)=\mathcal{M}\left(X_{i}\right)$ and $\left|X_{i}\right|$ and $\left|H_{i}\right|$ have the same prime divisors. But then, by Lemma $17, \mathcal{M}(G) \cong \mathcal{M}\left(H_{1}\right) \times \cdots \times \mathcal{M}\left(H_{u}\right) \cong \mathcal{M}\left(X_{1}\right) \times \cdots \times \mathcal{M}\left(X_{u}\right) \cong$ $\mathcal{M}\left(X_{1} \times \cdots \times X_{u}\right)$.

\section{REFERENCES}

1. H. Ahmadi and B. Taeri, A graph related to the join of subgroups of a finite group, Rend. Semin. Mat. Univ. Padova 131 (2014), 281-292.

2. A. Ballester-Bolinches and L. M. Ezquerro, Classes of finite groups, in Mathematics and Its Applications, vol. 584 (Springer, Dordrecht, 2006).

3. R. Baer, The significance of the system of subgroups for the structure of the group, Amer. J. Math. 61(1) (1939), 1-44.

4. J. Bray, D. Holt and C. Roney-Dougal, The maximal subgroups of the low-dimensional finite classical groups. With a foreword by Martin Liebeck. London Mathematical Society Lecture Note Series, vol. 407 (Cambridge University Press, Cambridge, 2013).

5. T. Burness, On base sizes for almost simple primitive groups, J. Algebra 516 (2018), 38-74.

6. T. Burness, M. Garonzi and A. Lucchini, On the minimal dimension of a finite simple group. With an appendex by T. C. Burnes and R. M. Guralnick, J. Combin. Theory Ser. A 171 (2020), 105175 .

7. T. Burness, R. Guralnick and J. Saxl, On base sizes for symmetric groups, Bull. Lond. Math. Soc. 43(2) (2011), 386-391.

8. T. Burness, M. Liebeck and A. Shalev, Base sizes for simple groups and a conjecture of Cameron, Proc. Lond. Math. Soc. (3) 98(1) (2009), 116-162.

9. M. Garonzi and A. Lucchini, Maximal irredundant families of minimal size in the alternating group, Arch. Math. (Basel) 113(2) (2019), 119-126.

10. W. Gaschütz, Die Eulersche Funktion endlicher auflösbarer Gruppen, Illinois J. Math. 3 (1959), 469-476.

11. S. Guest and P. Spiga, Finite primitive groups and regular orbits of group elements, Trans. Amer. Math. Soc. 369(2) (2017), 997-1024. 
12. P. Hall, The eulerian functions of a group, Quart. J. Math. 7 (1936), 134-151.

13. T. Hawkes, I. M. Isaacs and M. Özaydin, On the Möbius function of a finite group, Rocky Mountain J. Math. 19(4) (1989), 1003-1034.

14. P. Kleidman and M. Liebeck, The subgroup structure of the finite classical groups. London Mathematical Society Lecture Note Series, 129. (Cambridge University Press, Cambridge, 1990).

15. C. Kratzer and J. Thvenaz, Fonction de Möbius d'un groupe fini et anneau de Burnside, Comment. Math. Helv. 59(3) (1984), 425-438.

16. M. Liebeck, C. Praeger and J. Saxl, On the O'Nan-Scott theorem for finite primitive permutation groups, J. Austral. Math. Soc. Ser. A 44(3) (1988), 389-396.

17. R. Schmidt, Subgroup lattices of groups, De Gruyter Expositions in Mathematics, vol. 14 (Walter de Gruyter, Berlin, 1994).

18. M. Suzuki, Group theory I, Grundlehren der Mathematischen Wissenschaften vol. 247 (Springer-Verlag, Berlin, 1982).

19. T. R. Wolf, Large orbits of supersolvable linear groups, J. Algebra 215(1) (1999), 235-247. 